\title{
Brevilin A, a Natural Sesquiterpene Lactone Inhibited the Growth of Triple-Negative Breast Cancer Cells via Akt/mTOR and STAT3 Signaling Pathways
}

This article was published in the following Dove Press journal: OncoTargets and Therapy

\author{
Zhao Qu' \\ Yushan Lin' \\ Daniel Kam-Wah Mok',2 \\ Qingya Bian ${ }^{3}$ \\ William Chi-Shing Tai ${ }^{1,2,4}$ \\ Sibao Chen ${ }^{1-3}$ \\ 'State Key Laboratory of Chinese \\ Medicine and Molecular Pharmacology \\ (Incubation), Shenzhen Research \\ Institute, The Hong Kong Polytechnic \\ University, Shenzhen, Guangdong \\ 5 18057, People's Republic of China; \\ ${ }^{2}$ Department of Applied Biology \& \\ Chemical Technology, The Hong Kong \\ Polytechnic University, Hung Hom, \\ Hong Kong, S.A.R, People's Republic of \\ China; ${ }^{3}$ Institute of Medicinal Plant \\ Development, Chinese Academy of \\ Medical Sciences and Peking Union \\ Medical College, Beijing 100193, People's \\ Republic of China; ${ }^{4}$ Caritas Medical \\ Centre, Department of Clinical \\ Pathology, Sham Shui Po, Hong Kong, S.A. \\ R, People's Republic of China
}

Purpose: Triple-negative breast cancer (TNBC) is a a breast cancer subtype characterized by a lack of estrogen receptor, progesterone receptor and human epidermal growth receptor 2 and is associated with poorer prognoses when compared to other breast cancers. Thus, novel anti-cancer agents with high efficacy are urgently needed. Brevilin A (BA), a natural sesquiterpene lactone, has been reported to exhibit anti-cancer effects. However, the effects of BA on TNBC have not yet been demonstrated. In this study, we investigated the antiTNBC effects and the underlying mechanism of BA, in vitro and in vivo.

Methods: Two TNBC cell lines and a xenograft mouse model were employed to assess the effects of BA. Cell viability was detected by MTT assay. Cell cycle status and apoptosis were evaluated by flow cytometry. Cell migration was measured by wound-healing assay. Protein expression was measured by Western blotting analysis. The in vivo anti-cancer activity of BA was assessed in orthotopic tumor xenograft mice.

Results: BA significantly inhibited the growth of TNBC cells in a dose- and time-dependent manner via induction of cell cycle arrest at G2/M phase arrest and apoptosis. BA also inhibited tumor cell migration. BA significantly downregulated the expression of Akt, mTOR, Stat 3 and their phosphorylation, and thus inhibiting the activation of the Akt/mTOR and STAT3 signaling pathways. Furthermore, oral administration of BA at 25 or $50 \mathrm{mg} / \mathrm{kg}$ leads to significant inhibition of tumor growth and proliferation in tumor xenograft model mice.

Conclusion: BA significantly inhibited the growth and migration of TNBC cells, and induced cell cycle arrest and apoptosis. These inhibitory effects were associated with the suppression of the Akt/mTOR and Stat3 signal pathways. Based on our findings, BA possesses a promising candidate for development as an anti-cancer therapeutic drug against TNBC.

Keywords: anticancer, apoptosis, cell cycle arrest, migration, cancer therapy

\section{Introduction}

Breast cancer is the most common malignancy in women worldwide. According to the World Health Organization International Agency for Research on Cancer, there were approximately 2.1 million newly diagnosed female breast cancer cases in 2018, accounting for almost 1 in 4 cancer cases amongst women worldwide. ${ }^{1}$ Triple-negative breast cancer (TNBC) is characterized by a lack of estrogen receptor (ER), progesterone receptor (PR) and human epidermal growth receptor 2 (HER2), and accounts for approximately $15 \%-20 \%$ of all breast cancer cases. ${ }^{2}$ TNBC tumors do not respond well to endocrine and anti-HER2 therapies, and systemic treatment is limited to cytotoxic chemotherapy. ${ }^{3}$ Furthermore, compared to other breast cancer subtypes, TNBC has a higher risk of relapse and metastasis,
Correspondence: William Chi-Shing Tai;

State Key Laboratory of Chinese Medicine and Molecular Pharmacology (Incubation), Shenzhen Research Institute, The Hong Kong Polytechnic University, Shenzhen, Guangdong 518057, People's

Republic of China

Tel +86-0755-26737182

Fax +86-0755-26972852

Email william-cs.tai@polyu.edu.hk;

sibao.chen@polyu.edu.hk 
a shorter progression-free survival (PFS), and poorer overall survival. ${ }^{4}$ Therefore, new therapeutic drugs are needed in the fight against TNBC.

Brevilin A (BA), namely [(1S,3aR,5R,5aR,8aR,9S, 9aR)-1,5,8a-trimethyl-2,8-dioxo-3a,4,5,5a,9,9a-hexahydro

-1H-azuleno [6,5-b] furan-9-yl] (Z)-2-methylbut-2-enoate (Figure 1), is a sesquiterpene lactone extracted from Centipeda minima (L.) A. Br. et Aschers., and has been reported to exhibit anti-viral and anti-cancer effects, ${ }^{5,6}$ in cancers such as colon carcinoma, glioblastoma and nasopharyngeal carcinoma. ${ }^{7-9}$ However, the effects of BA on TNBC have not yet been demonstrated. Thus, in this study, we investigated the in vitro and in vivo anti-cancer effects of BA in TNBC.

The activation of the PI3K/Akt/mTOR pathway leads to uncontrolled of cell growth and survival, ultimately resulting in competitive growth advantage, metastatic competence, angiogenesis, and therapy resistance. ${ }^{10}$ STAT3 plays crucial roles in cell proliferation, survival and differentiation, and has been shown to be persistently activated in a high percentage of malignancies, including TNBC. ${ }^{11,12}$ Therefore, the involvement of the Akt/mTOR and STAT3 signaling pathways in the molecular mechanisms underlying the effects of BA were investigated.

\section{Materials and Methods}

\section{Reagents}

Brevilin A (purity $>98 \%$ ) was purchased from Jiangsu Yongjian Pharmaceutical Co., Ltd. (Jiangsu, China). Annexin V-FITC apoptosis detection kit and cell cycle analysis kit were

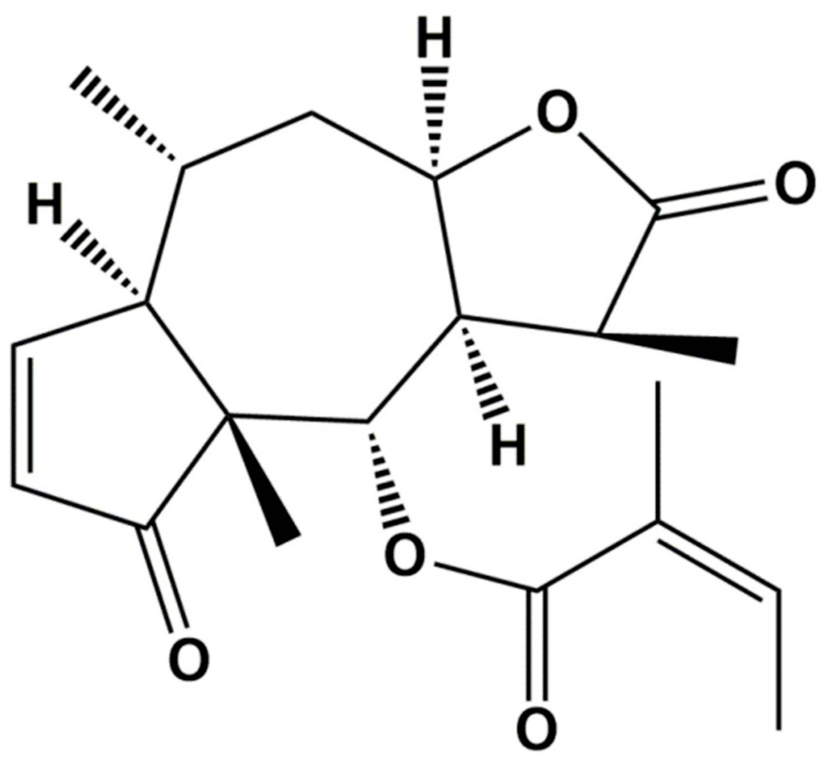

Figure I Chemical structure of BA. obtained from Beyotime Biotechnology (Beyotime, Shanghai, China). Primary and secondary antibodies were purchased from Cell Signaling Technology Inc. (Beverly, MA, USA).

\section{Cell Culture}

Human TNBC cell lines MDA-MB-231 and MDA-MB -468 and the non-TNBC cell line MCF7 were purchased from the American Type Culture Collection (ATCC, Manassas, VA, USA) and maintained in DMEM supplemented with $10 \%$ heat-inactivated FBS and penicillin (100 $\mathrm{U} / \mathrm{mL})$-streptomycin $(100 \mu \mathrm{g} / \mathrm{mL})$ at $37^{\circ} \mathrm{C}$ in a humidified atmosphere containing $5 \% \mathrm{CO}_{2}$ and $95 \%$ air.

\section{Cell Viability Assay}

Cells were seeded in a 96-well plate at a density of $5 \times 10^{4}$ (MDA-MB-231 and MCF7) or $1 \times 10^{5}$ (MDA-MB-468) cells $/ \mathrm{mL}$ for $24 \mathrm{~h}$, and then treated with varying concentrations of BA for 24,48 , or $72 \mathrm{~h} .20 \mu \mathrm{L}$ MTT $(2.5 \mathrm{mg} / \mathrm{mL})$ was added to the medium in each well and incubated for $4 \mathrm{~h} .100$ $\mu \mathrm{L}$ DMSO was added to dissolve formazan after discarding culture media. After shaking for $10 \mathrm{~min}$, the optical density (OD) was measured using a Biotek Synergy H1 microplate reader (Biotek, USA). $\mathrm{IC}_{50}$ values were estimated using Graphpad Prism 5 (Graph-Pad Software, CA, USA).

\section{Cell Cycle Analysis}

MDA-MB-231 and MDA-MB-468 cells were seeded at $2 \times$ $10^{5}$ cells per well in a 6-well plate and treated with BA for 24 or $48 \mathrm{~h}$. Cells were fixed with ice-cold $70 \%$ ethanol at $4^{\circ} \mathrm{C}$ overnight, then rinsed with $\mathrm{PBS}$ and incubated with propidium iodide (PI) for $30 \mathrm{~min}$ at $37^{\circ} \mathrm{C}$ in the dark. Flow cytometric cell analysis was performed using a BD AccuriC6 flow cytometry system (Becton Dickson ImmunocytometrySystems, San Jose, CA, USA) and the results were analyzed using ModFit LT 5.0 (Verity Software House, ME, USA)

\section{Annexin V-FITC Apoptosis Assay}

MDA-MB-231 and MDA-MB-468 cells were seeded at $2 \times$ $10^{5}$ cells per well in a 6-well plate and treated with BA for 24 or $48 \mathrm{~h}$. After $0.25 \%$ trypsin (without EDTA) treatment, cells were stained with FITC-labeled AnnexinV and PI at room temperature for $20 \mathrm{~min}$ in the dark. Stained cells were placed on ice in the dark and immediately assessed using a CytoFLEX flow cytometer (Beckman Coulter, Brea, CA, USA).

\section{Morphological Observation}

Cells $\left(1 \times 10^{5}\right.$ cells $\left./ \mathrm{mL}\right)$ were seeded into 6-well plates and treated with BA for $24 \mathrm{~h}$. To visualize DNA, cells 
were washed twice with PBS, fixed with $4 \%$ paraformaldehyde for $20 \mathrm{~min}$, stained with Hoechst 33,342 (10 $\mu \mathrm{g} / \mathrm{mL}$ ) for $15 \mathrm{~min}$ in the dark, and then visualized under an inverted fluorescence microscope (Nikon, Tokyo, Japan).

\section{Wound-Healing Assay}

MDA-MB-231 cells were seeded at $4 \times 10^{4}$ cells/well into both chamber of a $35 \mathrm{~mm}$ high $\mu$-dish with culture inserts (ibidi, Germany) for $24 \mathrm{~h}$. Cells were treated with $0,2.5,5,7.5$, and $10 \mu \mathrm{M}$ BA after removal of culture inserts. The process of wound healing was observed under a light microscope. Three randomly images were acquired at $0,6,12,24$, and $48 \mathrm{~h}$ after BA treatment. The percentage wound area was calculated as follows: scratch open area $(\%)=($ remaining wound area/original wound area vehicle) $\times 100$.

\section{Western Blot}

Cells were lysed in RIPA buffer (Cell signaling technology, Beverly, MA, USA) supplemented with $1 \mathrm{mM}$ PMSF (Sigma), protease inhibitor (Roche) and phosphatase inhibitor (Roche). Protein concentrations were determined by Pierce $^{\mathrm{TM}}$ BCA Protein Assay (Thermo Scientific). Protein extracts $(10 \mu \mathrm{g}$ per well) were separated by SDS-PAGE and then transferred to a PVDF membrane (BioRad). The blots were then blocked in 5\% non-fat skim milk in TBST buffer and probed using primary antibodies in 5\% BSA in TBST buffer overnight. After washing with TBST buffer, corresponding goat anti-rabbit and goat anti-mouse HRPconjugated secondary antibodies were added. Protein bands were visualized using ECL Western blotting detection reagents (Tanon). Protein expression levels were quantified by scanning autoradiogram and densitometry (Quantity One, Bio-Rad).

\section{Animals}

6-week-old female BALB/c nude mice were purchased from Beijing Vital River Laboratory Animal Technology Co., Ltd. (Beijing China). Animals were housed in an SPF grade animal facility with food and water available $a d$ libitum. All animal experimental procedures were carried out in compliance with the Institutional Guidelines and Animal Ordinance of the Department of Health and approved by the Hong Kong Polytechnic University Animal Subjects Ethics Sub-committee.

\section{Tumor Xenograft Study}

MDA-MB-231 cells $\left(5 \times 10^{6}\right)$ were suspended in $100 \mu \mathrm{L}$ medium and inoculated in the fourth mammary fat pad. After two weeks inoculation, when average tumor volumes reached $70 \mathrm{~mm}^{3}$, mice were randomly divided into four groups, each receiving different treatment. As a control, mice were treated with $0.5 \%$ carboxymethyl cellulose (CMC) containing $1 \%$ Tween-80. For BA treatment groups, mice were treated orally with BA at 25 or $50 \mathrm{mg} / \mathrm{kg}$ /day (Tween- 80 was added to BA and then mixed with $0.5 \% \mathrm{CMC}$ ). As a positive control, mice were injected intraperitoneally with $10 \mathrm{mg} / \mathrm{kg}$ of docetaxel once a week. Every two or three days, body weights and tumor sizes were measured. Tumor volumes were calculated using the formula $\left(\mathrm{L} \times \mathrm{W}^{2}\right) / 2 \mathrm{~mm}^{3}$ ( $\mathrm{L}=$ length; $\mathrm{W}=$ width). All mice were sacrificed on day 22 treatment, and their tumors and vital organs were harvested and weighed.

\section{Histological Analyses}

Tumors were fixed in $10 \%$ neutral buffered formalin and, embedded with paraffin. $5 \mu \mathrm{m}$ sections were cut and stained with hematoxylin and eosin (H\&E). Immunohistochemical analyses for Ki67 were performed using SPlink Detection Kits (ZSGB-BIO, Beijing, China). Stained sections were examined using a Nikon Observer microscope.

\section{Statistical Analyses}

All data are expressed as means \pm S.E.M., statistical differences between groups were analysed by one-way analysis of variance (ANOVA) followed by LSD post hoc or Dunnett's tests. Differences were considered significant at $\mathrm{P}$ values of $<0.05$.

\section{Results \\ BA Inhibited Breast Cancer Cell Proliferation}

To evaluate the in vitro anti-proliferative activity of brevilin A in breast cancer, MDA-MB-231, MDA-MB-468, and MCF7 cells were treated with brevilin A for 24,48 , and $72 \mathrm{~h}$. Results from MTT assays showed that brevilin A significantly inhibited the growth of breast cancer cells in a dose- and time-dependent manner (Figure 2A-C, Table 1). As shown in Table 1, the $\mathrm{IC}_{50}$ values of brevilin A in MDAMB-231 were 13.31, 5.723, and $3.764 \mu \mathrm{M}$ at 24, 48, and 72 $\mathrm{h}$ of treatment respectively. In MDA-MB-468, $\mathrm{IC}_{50}$ values were $14.46,4.089$, and $3.034 \mu \mathrm{M}$ respectively at 24,48 , and $72 \mathrm{~h}$ of treatment. For ER- and PR-overexpressing MCF7, $\mathrm{IC}_{50}$ values were $10.62,9.475$, and $6.478 \mu \mathrm{M}$ respectively at 
A

MDA-MB-231

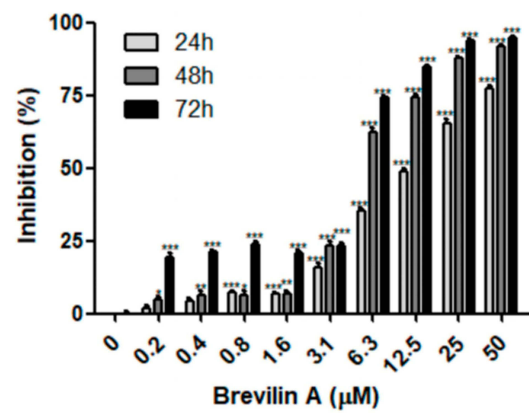

B

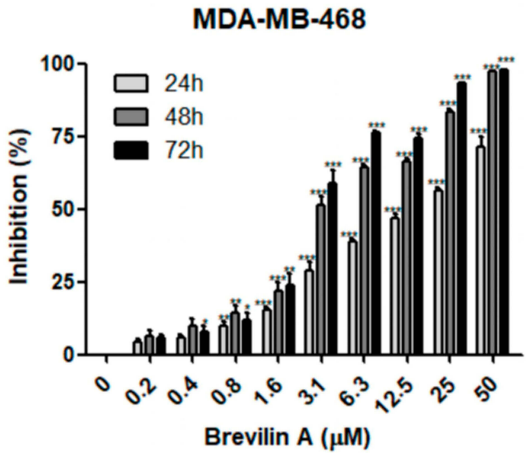

C

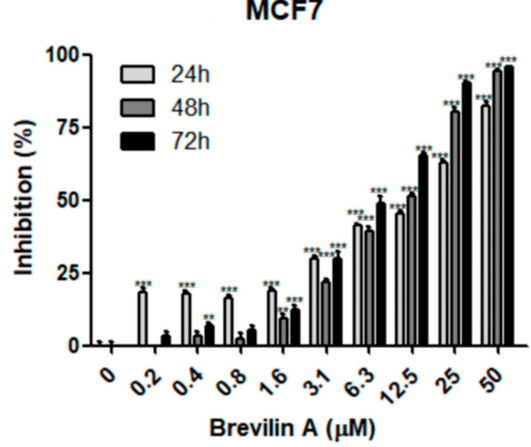

Figure 2 BA inhibited breast cancer cell proliferation. Cell viability of (A) MDA-MB-23I, (B) MDA-MB-468, and (C) MCF7 was measured by MTT assay after BA treatment at the indicated concentrations for 24,48 , and $72 \mathrm{~h}$. Data presented as means $\pm \mathrm{SEM}$ from three independent experiments. $* \mathrm{P}<0.05$, $* * \mathrm{P}<0.0 \mathrm{I}$, $* * * \mathrm{P}<0.00 \mathrm{I}$, compared to control.

24,48 , and $72 \mathrm{~h}$ of treatment. The time-dependent inhibition of growth by brevilin A in the MDA-MB-231 and MDA-MB -468 TNBC cell lines was much more pronounced when compared to MCF7. The $\mathrm{IC}_{50}$ values of brevilin $\mathrm{A}$ in MCF7 were approximately twice that of MDA-MB-231 and MDA-MB-468 for 48 and $72 \mathrm{~h}$ treatments.

\section{BA Induced G2/M Arrest in TNBC Cells}

To investigate the mechanisms underlying the proliferative inhibition of TNBC cells by BA, we examined its effects on the cell cycle, via flow cytometry analysis. MDA-MB-231 and MDA-MB-468 cells were treated with BA for 24 or $48 \mathrm{~h}$ and then stained with propidium iodide (PI). Results showed that BA significantly increased the percentage of cells in the $G 2 / M$ phase from at concentrations as low as $5 \mu \mathrm{M}$ (Figure 3A and $\mathrm{B})$. We further examined the protein expression of key regulators of the cell cycle. BA decreased the expression of Cyclin D1, Cyclin D3, CDK6, cdc2, and Cyclin B1 in MDA-MB-231 cells (Figure 3C). In MDA-MB-468 cells, brevilin A decreased the expression of Cyclin D3 and cdc2, and upregulated the expression of Cyclin B1 (Figure 3D).

\section{BA Induced Apoptosis in TNBC Cells}

To test whether BA induced apoptosis, we measured apoptosis via AnnexinV-FITC/PI staining and flow

Table I IC 50 Values $(\mu \mathrm{M})$ of Brevilin a in Human Breast Cancer Cell Lines

\begin{tabular}{|l|l|l|l|}
\hline Cell Line & $\mathbf{2 4} \mathbf{h}$ & $\mathbf{4 8} \mathbf{h}$ & $\mathbf{7 2} \mathbf{~}$ \\
\hline MDA-MB-23I & 13.31 & 5.723 & 3.764 \\
MDA-MB-468 & 14.46 & 4.089 & 3.034 \\
MCF7 & 10.62 & 9.475 & 6.478 \\
\hline
\end{tabular}

cytometry in TNBC cells. 24 or $48 \mathrm{~h}$ BA treatment could induce apoptosis of MDA-MB-231, mainly at the late stage (Figure 4A). In MDA-MB-468 cells, BA induced both early and late apoptosis (Figure 4B). To observe the morphological changes, we stained MDAMB-231 and MDA-MB-468 cells with Hoechst 33,342 after $24 \mathrm{~h}$ BA treatment. As shown in Figure 4C, BAtreated cells exhibited high chromatin condensation and apoptotic body formation, characteristics associated with apoptosis. Furthermore, BA down-regulated the expression of the apoptosis-associated protein, Caspase 3, and increased levels of cleaved Caspase 3 (Figure 4D).

\section{BA Inhibited Migration of MDA-MB-23 I Cells}

Compared to other types of breast cancers, TNBC exhibits a highly invasive characteristic. Therefore, we investigated the effects of BA on the invasive potential of MDA-MB-231 cells using a wound-healing assay. Results demonstrated that BA suppressed cell migration in a dose-dependent manner. After 24 and $48 \mathrm{~h}$, treatment with BA $(5,7.5$ and $10 \mu \mathrm{M})$ significantly inhibited cell migration (Figure 5).

\section{BA Downregulated Akt/mTOR Signaling in TNBC Cells}

Activation of the AKT/mTOR signaling pathway plays a significant role in controlling cell growth, proliferation and metastasis. ${ }^{13}$ Therefore, we examined key proteins of the AKT/mTOR signaling pathway in MDA-MB-231 and MDA-MB-468 cells. As shown in Figure 6, BA significantly downregulated the expression of Akt, 

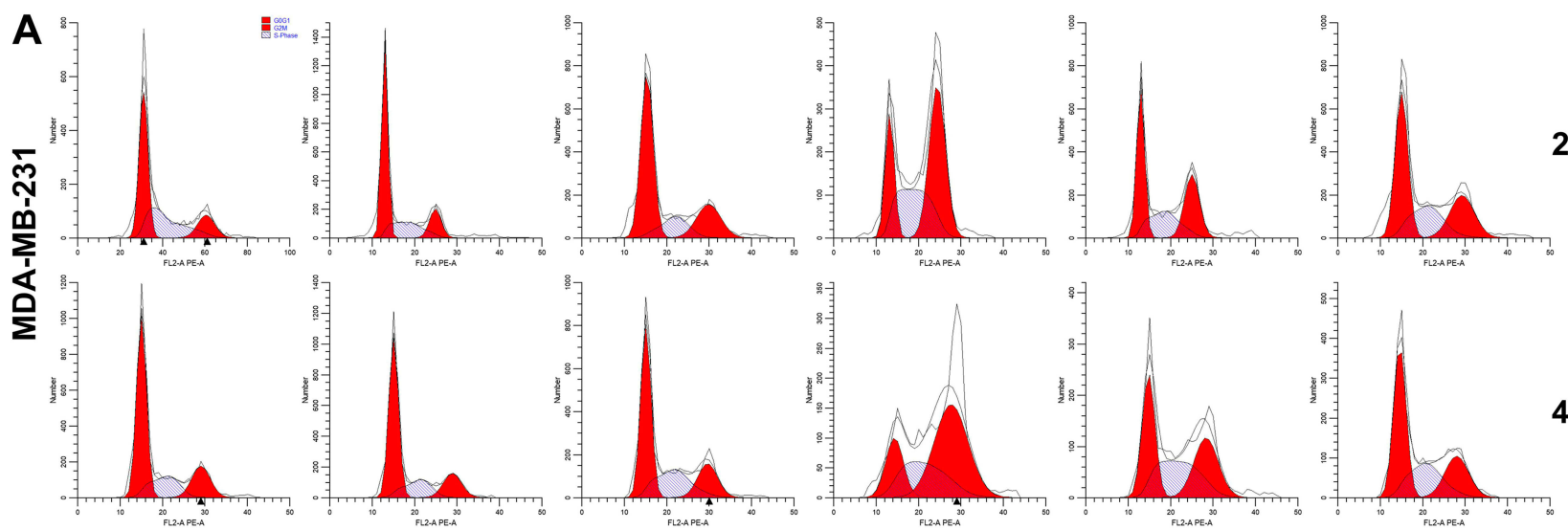

$24 h$
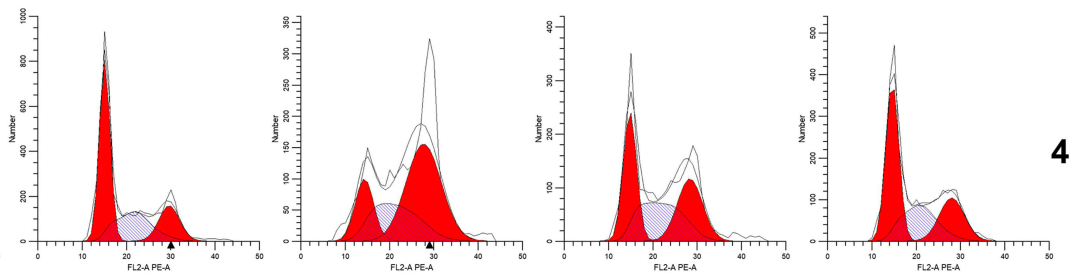

$48 h$

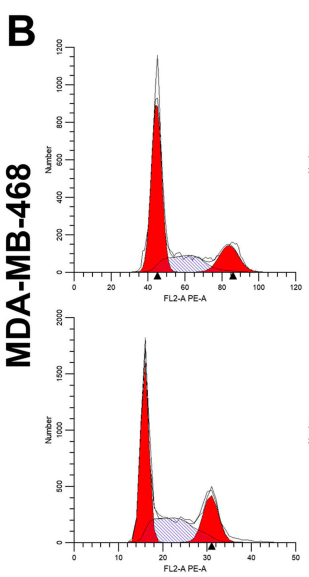

0
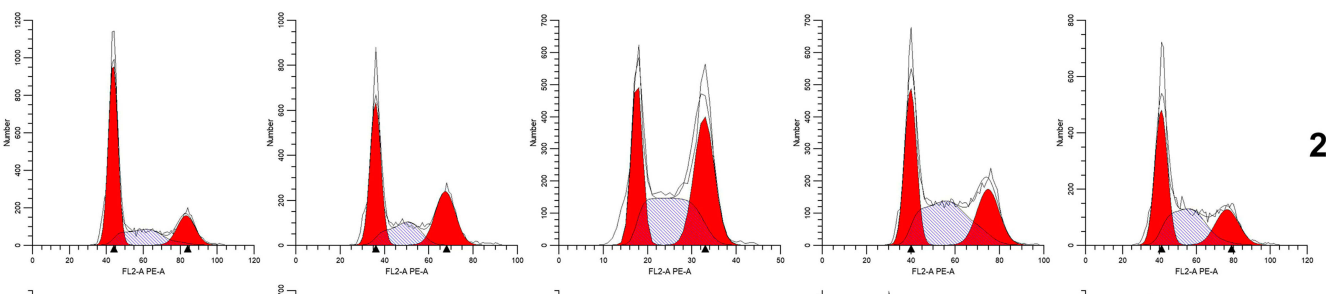

$24 \mathrm{~h}$
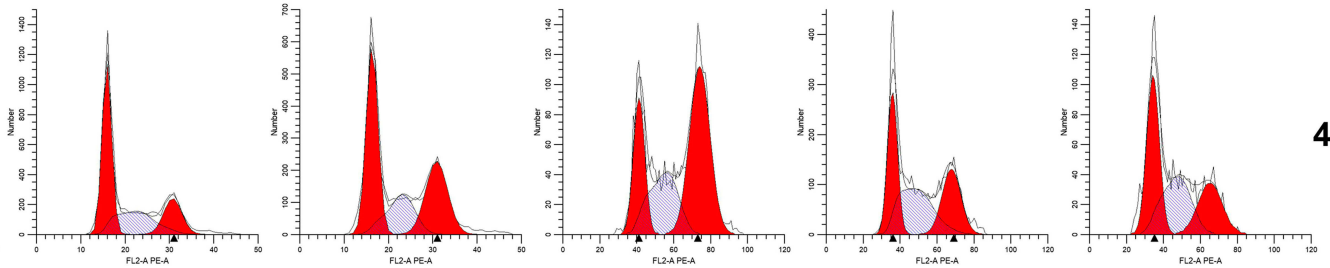

$48 h$

C

MDA-MB-231

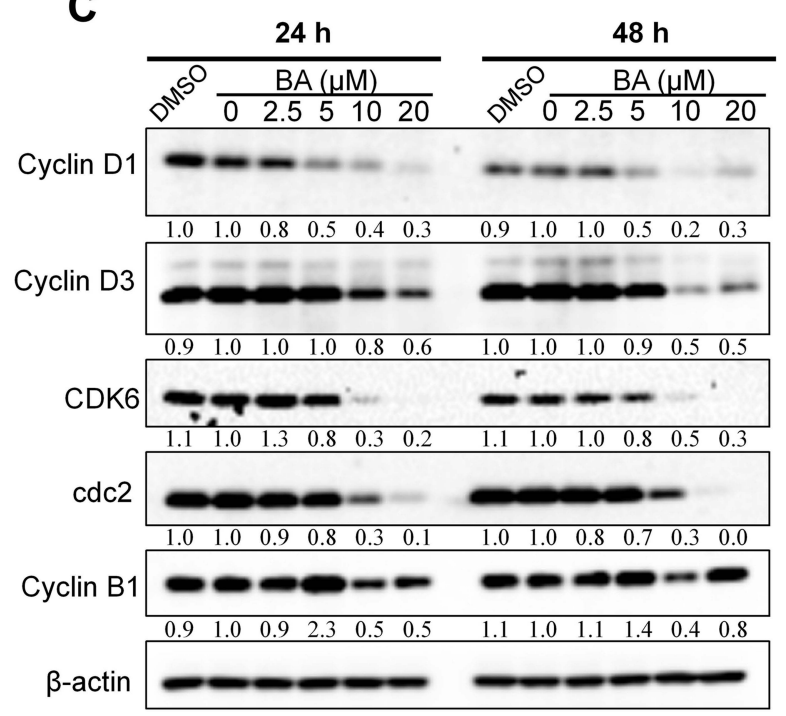

MDA-MB-468

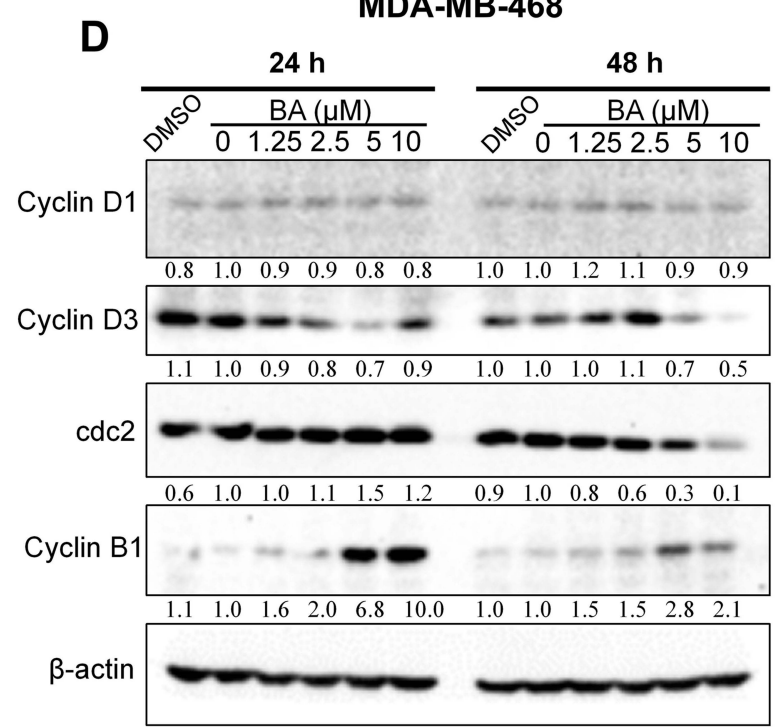

Figure 3 BA induced cell cycle arrest in TNBC. (A) MDA-MB-23I and (B) MDA-MB-468 cells were treated with BA for 24 or 48 h, stained with PI and effects on the cell cycle were analyzed by flow cytometry. (C) MDA-MB-23I and (D) MDA-MB-468 cells were treated with BA for 24 or 48 h, collected, and immunoblotted with the indicated antibodies. $\beta$-actin was used as an internal control. 

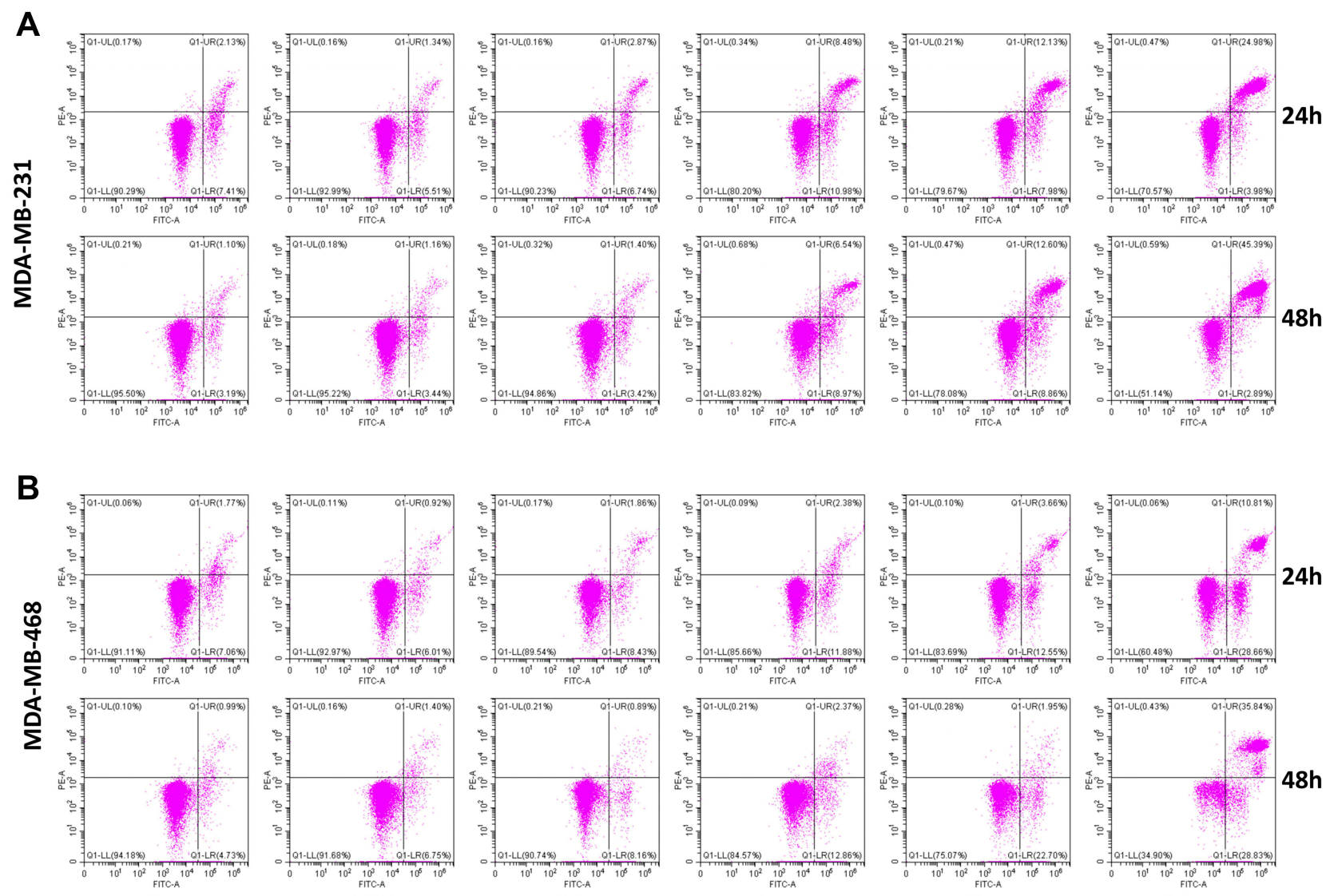

1.25
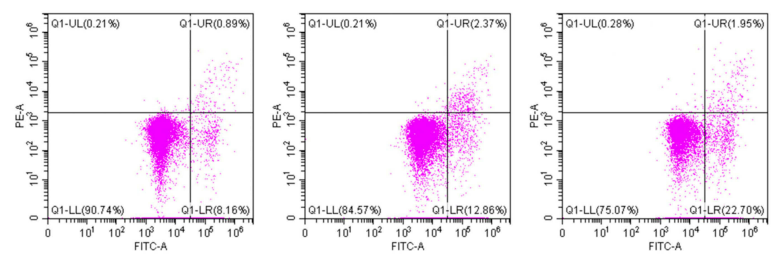

10

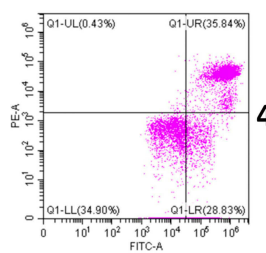

20

Brevilin A ( $\mu \mathrm{M})$

C
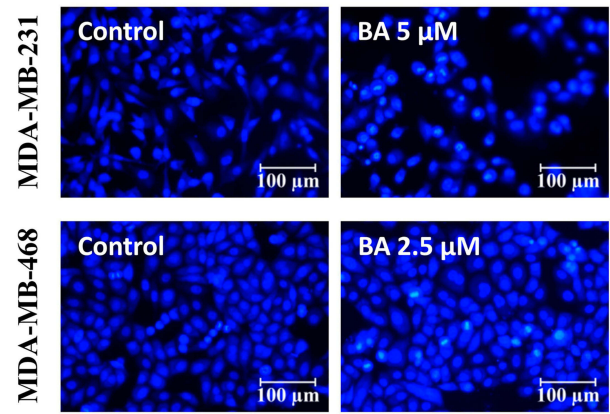

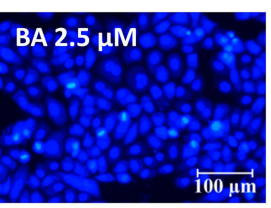

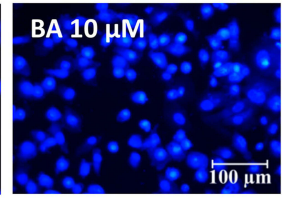

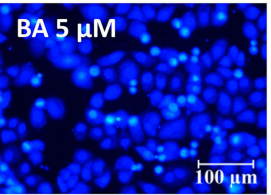

MDA-MB-231
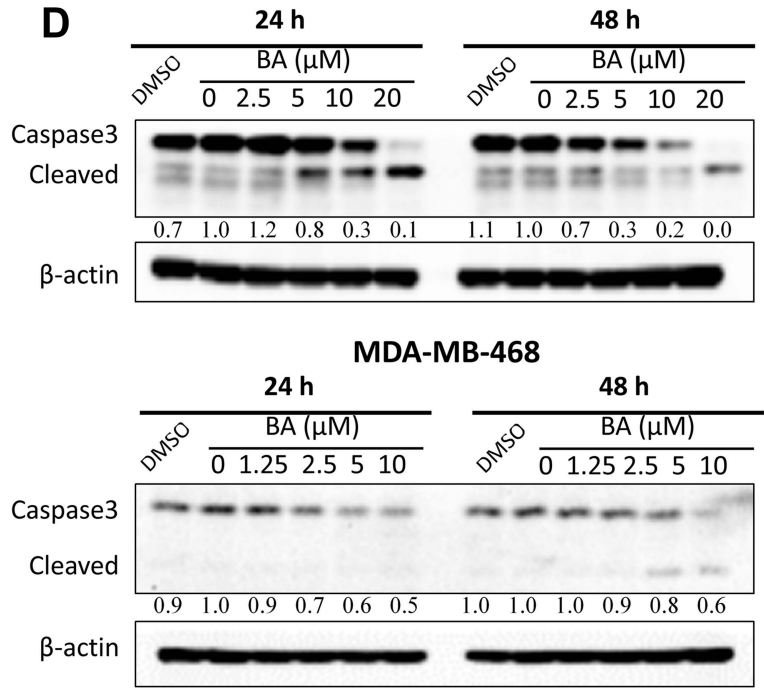

Figure 4 BA induced apoptosis in TNBC. (A) MDA-MB-23I and (B) MDA-MB-468 cells were treated with BA for 24 or 48 h, stained with Annexin V-FITC/PI and cell apoptosis was analyzed by flow cytometry. (C) MDA-MB-23I and MDA-MB-468 cells were treated with BA for 24 h, stained with Hoechst 33,342 and nuclear morphology was photographed with an inverted fluorescence microscope $(200 \mathrm{x})$. Scale bar, $100 \mu \mathrm{m}$. (D) MDA-MB-23I and MDA-MB-468 cells were treated with BA for 24 or $48 \mathrm{~h}$, collected, and immunoblotted with the indicated antibodies. $\beta$-actin was used as an internal control.

mTOR, and phosphorylated mTOR in MDA-MB-231 and MDA-MB-468 cells. BA also inhibited the expression of phosphorylated Akt in MDA-MB-468 cells. As the expression of phosphorylated Akt in MDA-MB-231 was low, the modulation of BA on pAkt was not very obvious in this cell line. 

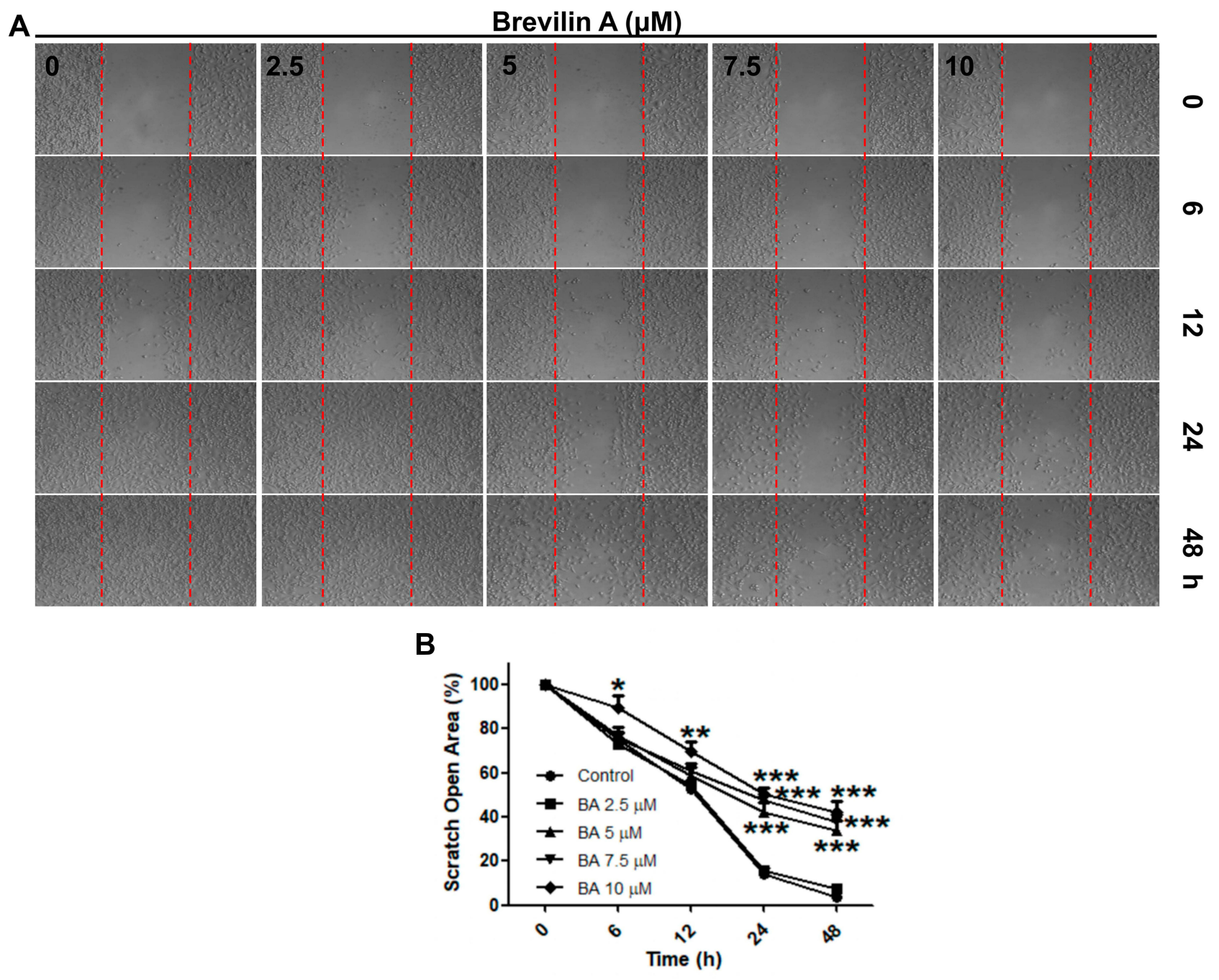

Figure 5 BA inhibited cell migration in MDA-MB-23I cells. (A) Cell migration was measured by wound-healing assay. (B) Quantification of scratch open area. Data are presented as means \pm SEM from three independent experiments. $* \mathrm{P}<0.05$, $* * P<0.01$, $* * * P<0.001$, compared to control.

\section{BA Downregulated STAT3 Signaling in TNBC Cells}

STAT3 is over-expressed and constitutively activated in numerous cancer types, including more than $40 \%$ of breast cancers, and it is also strongly associated with TNBC cell survival, proliferation, cell cycle progression, antiapoptosis, migration, and invasion. ${ }^{14,15}$ Therefore, expression levels of STAT3 were examined. As shown in Figure 7, in both MDA-MB-231 and MDA-MB-468 cells, BA significantly downregulated the expression of STAT3 and phosphorylated STAT3 in a dose-dependent manner.

\section{BA Inhibited MDA-MB-23I Xenograft Tumor Growth in vivo}

To investigate the in vivo anti-cancer effect of BA, we employed an MDA-MB-231 orthotopic xenograft mouse model. Mice received oral treatment of BA at low $(25 \mathrm{mg} /$ $\mathrm{kg})$ or high $(50 \mathrm{mg} / \mathrm{kg})$ doses, or i.p. injection of docetaxel as a positive control. After 22 days of treatment, mice were sacrificed. BA treatment suppressed tumor growth and endpoint tumor weight, without causing significant body weight loss (Figure 8A-D).

\section{BA Inhibited Cancer Cell Proliferation in vivo}

We next conducted H\&E staining of the tumor sections to examine the effects of BA on histology. Compared to the vehicle group, tumor cell density was greatly decreased after treatment with BA, in a magnitude similar to docetaxel (Figure 9). In addition, we examined the expression of cell proliferation marker Ki67. We found that the BA-treated group showed fewer Ki67 positive cells in than the vehicle group. 

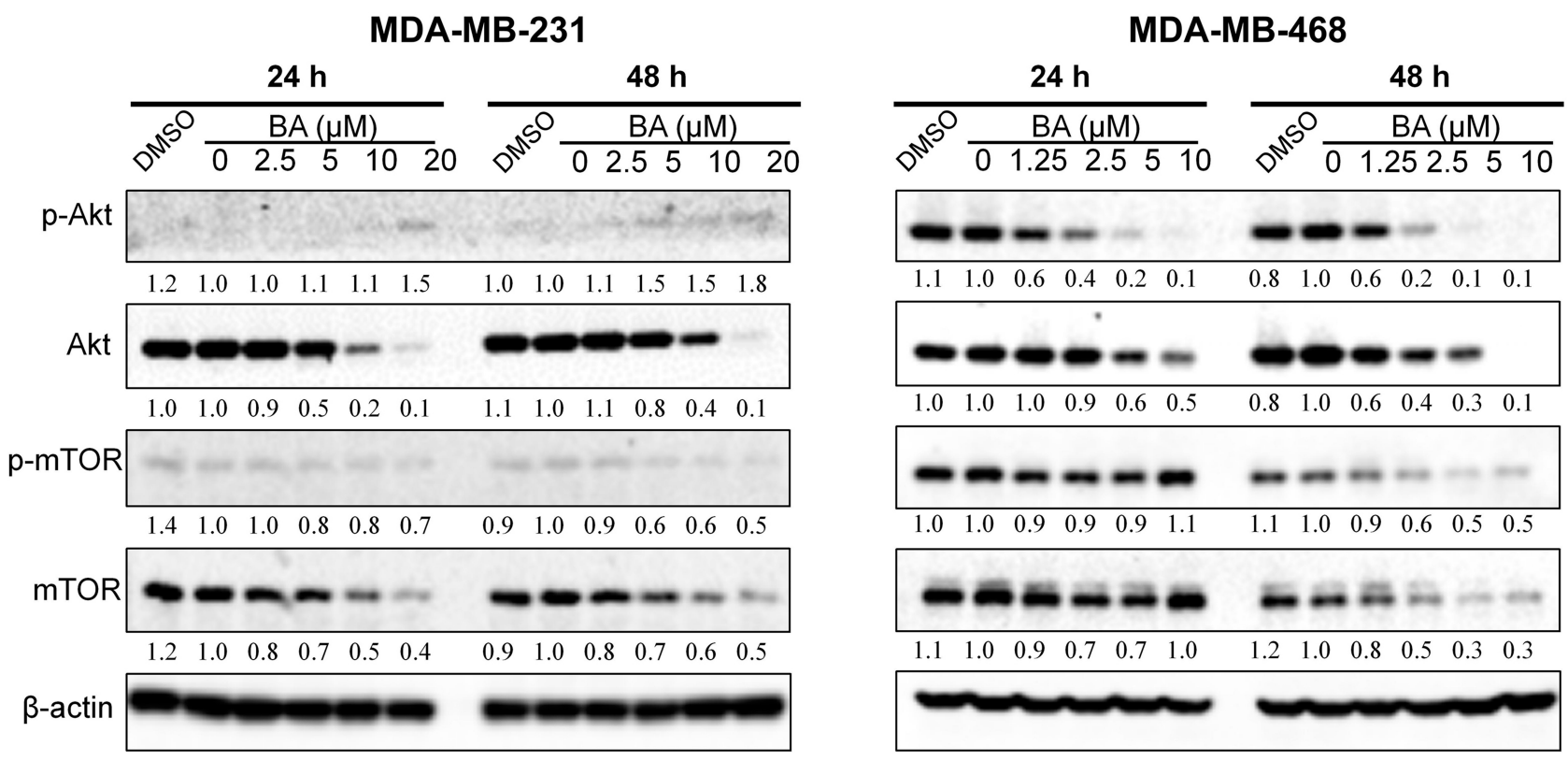

Figure 6 BA downregulated Akt/mTOR signaling in TNBC cells. MDA-MB-23I and MDA-MB-468 cells were treated with BA for 24 or 48 h, collected and immunoblotted with the indicated antibodies. $\beta$-actin was used as an internal control.

\section{Discussion}

In this study we demonstrated that BA had an in vitro and in vivo inhibitory effect on TNBC, via G2/M cell cycle arrest, induction of apoptosis, inhibition of cell migration, and suppression of the Akt/mTOR and STAT3 signaling pathways.

It has been reported that dysregulated CDKs result in unscheduled proliferation, and genomic and chromosomal instability in cancer cells. ${ }^{16}$ Cyclin D binding and activation of CDK4/6 initiates DNA synthesis during the G1 phase, and cdc2-cyclin B complexes are responsible for driving cells through mitosis. In this study, we found that
BA induced G2/M cell cycle arrest in MDA-MB-231 cells by downregulating the expression of cyclin D1, cyclin D3, CDK6, cdc2 and cyclin B1. BA also decreased the expression of Cyclin D3 and cdc2 in MDA-MB-468 cells. Cyclin B1 accumulates in the G2 phase and is rapidly degraded in anaphase of mitosis. ${ }^{17}$ The over-expression of cyclin B1 induced by BA suggested that MDA-MB-468 cells are arrested at the $\mathrm{G} 2$ phase.

Apoptosis is a major form of programmed cell death, including the mitochondrial mediated pathway (intrinsic) and the death receptor pathway (extrinsic). ${ }^{18}$ Caspase 3 is an effector caspase responsible for cleavage of
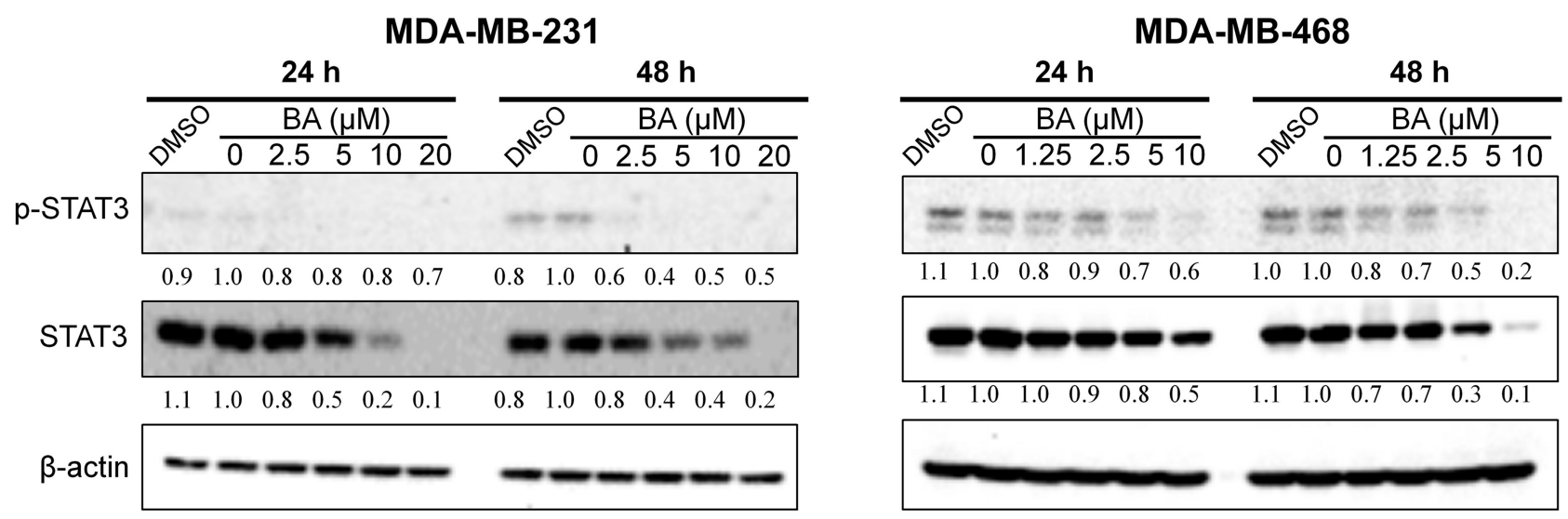

Figure 7 BA downregulated STAT3 signaling in TNBC cells. MDA-MB-23I and MDA-MB-468 cells were treated with BA for 24 or 48 h, collected and immunoblotted with the indicated antibodies. $\beta$-actin was used as an internal control. 
A

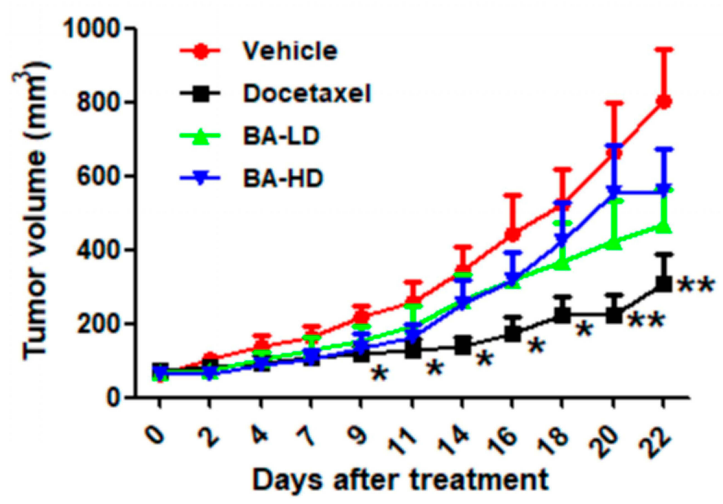

C

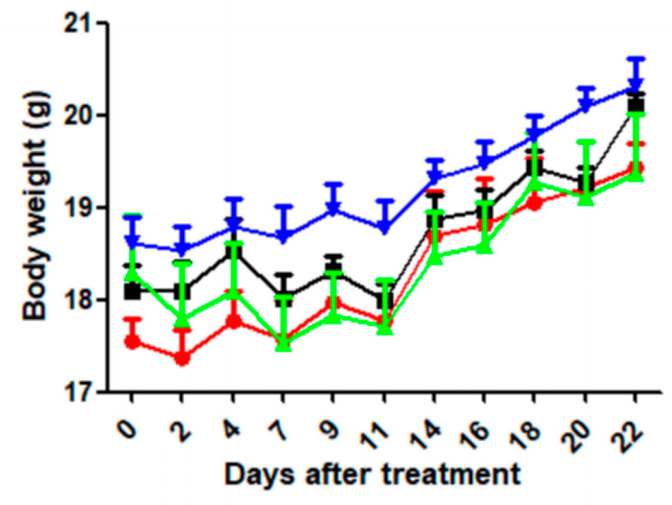

B

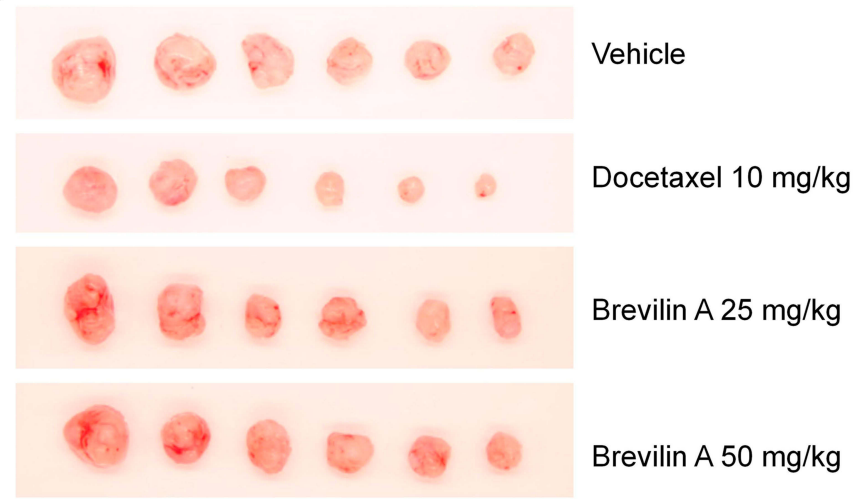

D

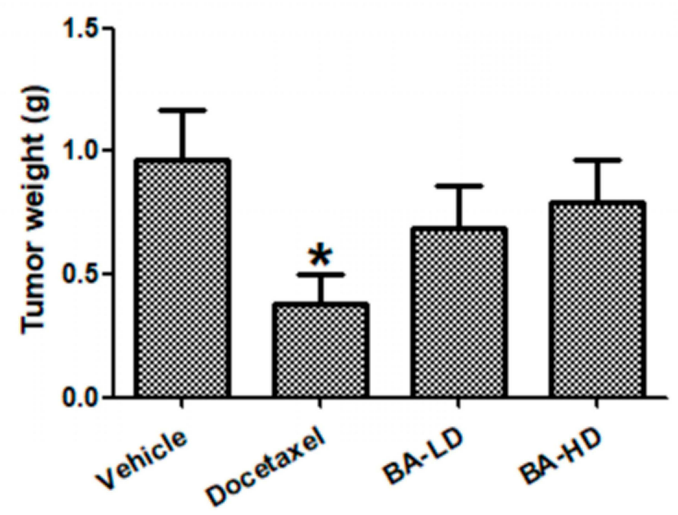

Figure 8 BA inhibited MDA-MB-23I xenograft tumor growth in vivo. Mammary fat pads of BALB/c nude mice were inoculated with MDA-MB-23I cells. When tumors grew to approximately $70 \mathrm{~mm}^{3}$, mice were randomized into groups and received different treatments: vehicle $(0.5 \% \mathrm{CMC}), \mathrm{BA}-\mathrm{LD}(25 \mathrm{mg} / \mathrm{kg})$, or BA-HD (50mg/kg) daily via oral gavage, or docetaxel $(10 \mathrm{mg} / \mathrm{kg})$ once a week via i.p. injection. The treatment period lasted for 22 days, after which mice were sacrificed and tumors were excised and weighed. (A) Average tumor volumes throughout the 22-day experimental period. (B) Representative photograph of tumors at experimental endpoint. (C) Mouse body weight throughout the 22-day experimental period. (D) Average tumor weight at experimental endpoint. Data are expressed as means $\pm \mathrm{SEM}$. $* \mathrm{P}<0.05$, $* * \mathrm{P}<0.0 \mathrm{I}$ compared to control.

proteasomal subunits. ${ }^{19}$ In this study, we found that BA induced apoptosis and the formation of apoptotic bodies via activation of Caspase 3 .
The AKT/mTOR pathway regulates cell proliferation and survival, and is found over-activated in more than $60 \%$ of TNBC patients. ${ }^{20}$ In addition, p-mTOR expression

H\&E
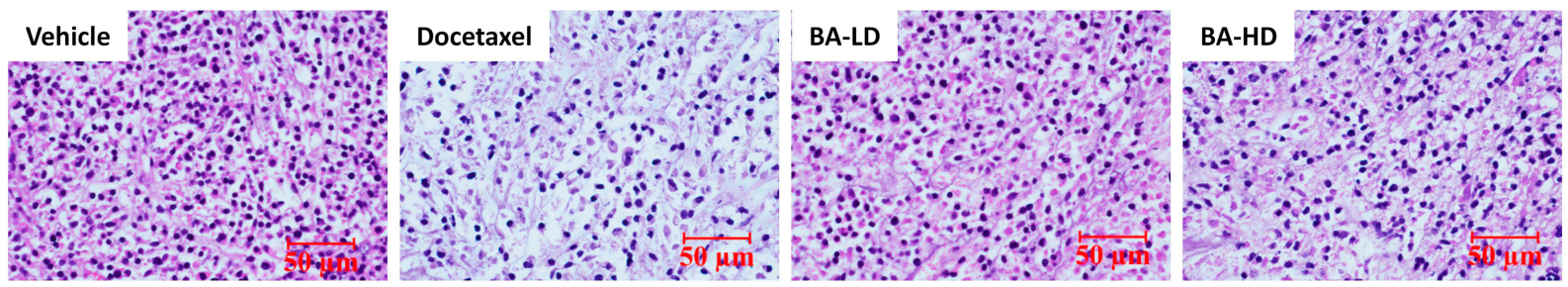

Ki67
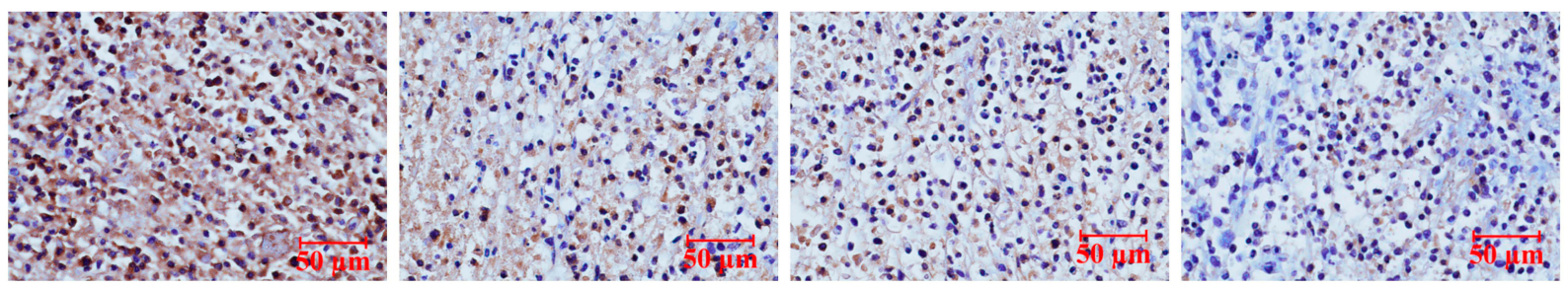

Figure 9 BA inhibited cancer cell proliferation in vivo. Tumors were stained with H\&E and Ki67. Representative images are shown (200 x). Scale bar, $50 \mu \mathrm{m}$. 
appears to be linked to poor prognosis in early-stage TNBC. $^{21}$ In recent years, the Akt/mTOR pathway has been considered as a therapeutic target for novel drug discovery and several compounds, such as PQR309, Ipatasertib, AZD5363, and temsirolimus are being clinically evaluated. ${ }^{22}$ Rapamycin, an mTOR inhibitor, is could effectively inhibit TNBC cell survival and induce cell cycle arrest but at high doses. ${ }^{23,24}$ In this study, we found that BA downregulated the expression of AKT and mTOR in TNBC cells and induced cell cycle arrest and apoptosis. Our results suggest that BA may exhibit improvements compared to rapamycin and inhibition of the AKT/mTOR pathway may be a potential mechanism for anti-TNBC activity.

STAT3 is constitutively activated in more than $40 \%$ of breast cancers, and is associated with initiation, progression, metastasis, chemoresistance, and immune evasion of TNBC. ${ }^{14,15}$ Several natural sesquiterpene lactones, such as alantolactone, parthenolide, and dehydrocostuslactone, exhibit anti-TNBC effects via downregulation of p-STAT3 expression. ${ }^{25}$ In our study, we found that BA inhibited the expression of STAT3 in MDA-MB-231 and MDA-MB-468 cells, indicating another potential route for the anti-TNBC activity of BA.

\section{Conclusion}

BA inhibited TNBC cell growth, induced G2/M cell cycle arrest and apoptosis, and suppressed cell migration. In addition, BA significantly prevented of the activation Akt/mTOR and STAT3 signaling pathways in TNBC cells. BA also exhibited in vivo anti-cancer effects in a nude mice xenograft model, without appreciable toxicity. Based on our findings, BA exhibits potential as a novel drug for the therapy of TNBC.

\section{Acknowledgments}

This work was supported by the Basic Research Foundation of the Shenzhen Science and Technology Innovation Committee (JCYJ20160229173844278, JCYJ20151030164022389), and the National Natural Science Foundation of China (No. 81872769) to SC, and the Health and Medical Research Fund, Food and Health Bureau, Hong Kong SAR (project no. 16172701) to WT.

\section{Disclosure}

The authors report no conflicts of interest in this work.

\section{References}

1. Bray F, Ferlay J, Soerjomataram I, et al. Global cancer statistics 2018: GLOBOCAN estimates of incidence and mortality worldwide for 36 cancers in 185 countries. CA Cancer J Clin. 2018;68 (6):394-424. doi:10.3322/caac.21492

2. Garrido-Castro AC, Lin NU, Polyak K. Insights into molecular classifications of triple-negative breast cancer: improving patient selection for treatment. Cancer Discov. 2019;9(2):176-198. doi:10.11 58/2159-8290.CD-18-1177

3. Shastry M, Yardley DA. Updates in the treatment of basal/triple-negative breast cancer. Curr Opin Obstet Gynecol. 2013;25(1):40-48. doi:10.1097/GCO.0b013e32835c1633

4. Lee KL, Kuo YC, Ho YS, et al. Triple-negative breast cancer: current understanding and future therapeutic breakthrough targeting cancer stemness. Cancers. 2019;11(9):pii: E1334. doi:10.3390/cancers1109 1334

5. Zhang X, Xia Y, Yang L, et al. Brevilin A, a sesquiterpene lactone, inhibits the replication of influenza a virus in vitro and in vivo. Viruses. 2019;11(9):pii: E835. doi:10.3390/v11090835

6. Chen X, Du Y, Nan J, et al. Brevilin A, a novel natural product, inhibits janus kinase activity and blocks STAT3 signaling in cancer cells. PLoS One. 2013;8(5):e63697. doi:10.1371/journal.pone.0063 697

7. You P, Wu H, Deng M, et al. Brevilin A induces apoptosis and autophagy of colon adenocarcinoma cell CT26 via mitochondrial pathway and PI3K/AKT/mTOR inactivation. Biomed Pharmacother. 2018;98:619-625. doi:10.1016/j.biopha.2017.12.057

8. Wang J, Li M, Cui X, et al. Brevilin A promotes oxidative stress and induces mitochondrial apoptosis in U87 glioblastoma cells. Onco Targets Ther. 2018;11:7031-7040. doi:10.2147/OTT.S179730

9. Liu R, Qu Z, Lin Y, et al. Brevilin A induces cell cycle arrest and apoptosis in nasopharyngeal carcinoma. Front Pharmacol. 2019;10: 594. doi:10.3389/fphar.2019.00594

10. Porta C, Paglino C, Mosca A. Targeting PI3K/Akt/mTOR signaling in cancer. Front Oncol. 2014;4:64. doi:10.3389/fonc.2014.00064

11. Laudisi F, Cherubini F, Monteleone G, et al. STAT3 interactors as potential therapeutic targets for cancer treatment. Int $J$ Mol Sci. 2018;19(6):pii: E1787. doi:10.3390/ijms 19061787

12. Ma JH, Qin L, Li X. Role of STAT3 signaling pathway in breast cancer. Cell Commun Signal. 2020;18(1):33. doi:10.1186/s12964$020-0527-\mathrm{z}$

13. Sharma VR, Gupta GK, Sharma AK, et al. PI3K/Akt/mTOR intracellular pathway and breast cancer: factors, mechanism and regulation. Curr Pharm Des. 2017;23(11):1633-1638. doi:10.2174/ 1381612823666161116125218

14. Banerjee K, Resat H. Constitutive activation of STAT3 in breast cancer cells: a review. Int $J$ Cancer. 2016;138(11):2570-2578. doi:10.1002/ijc.29923

15. Qin JJ, Yan L, Zhang J, et al. STAT3 as a potential therapeutic target in triple negative breast cancer: a systematic review. J Exp Clin Cancer Res. 2019;38(1):195. doi:10.1186/s13046-019-1206-z

16. Malumbres M, Barbacid M. Cell cycle, CDKs and cancer: a changing paradigm. Nat Rev Cancer. 2009;9(3):153-166. doi:10.1038/nrc2602

17. Widrow RJ, Rabinovitch PS, Cho K, et al. Separation of cells at different times within G2 and mitosis by cyclin B1 flow cytometry. Cytometry. 1997;27(3):250-254. doi:10.1002/(SICI)1097-0320(19970301)27:3<250::AID-CYTO6>3.0.CO;2-I

18. Reed JC. Mechanisms of apoptosis. Am J Pathol. 2000;157 (5):1415-1430. doi:10.1016/S0002-9440(10)64779-7

19. Sun XM, Butterworth M, MacFarlane M, et al. Caspase activation inhibits proteasome function during apoptosis. Mol Cell. 2004;14 (1):81-93. doi:10.1016/S1097-2765(04)00156-X

20. Kalimutho M, Parsons K, Mittal D, et al. Therapies for triple-negative breast cancer: combating a stubborn disease. Trends Pharmacol Sci. 2015;36(12):822-846. doi:10.1016/j.tips.2015.08.009 
21. Ueng SH, Chen SC, Chang YS, et al. Phosphorylated mTOR expression correlates with poor outcome in early-stage triple negative breast carcinomas. Int J Clin Exp Pathol. 2012;5(8):806-813.

22. Khan MA, Jain VK, Rizwanullah M, et al. PI3K/AKT/mTOR pathway inhibitors in triple-negative breast cancer: a review on drug discovery and future challenges. Drug Discov Today. 2019;24 (11):2181-2191. doi:10.1016/j.drudis.2019.09.001

23. Mukhopadhyay S, Frias MA, Chatterjee A, et al. The enigma of rapamycin dosage. Mol Cancer Ther. 2016;15(3):347-353. doi:10.1158/1535-7163.MCT-15-0720
24. Mukhopadhyay S, Chatterjee A, Kogan D, et al. 5-Aminoimidazole4-carboxamide-1- $\beta-4$-ribofuranoside (AICAR) enhances the efficacy of rapamycin in human cancer cells. Cell Cycle. 2015;14 (20):3331-3339. doi:10.1080/15384101.2015.1087623

25. Butturini E, Carcereri de Prati A, Boriero D, et al. Natural sesquiterpene lactones enhance chemosensitivity of tumor cells through redox regulation of STAT3 signaling. Oxid Med Cell Longev. 2019;2019:4568964. doi:10.1155/2019/4568964

\section{Publish your work in this journal}

OncoTargets and Therapy is an international, peer-reviewed, open access journal focusing on the pathological basis of all cancers, potential targets for therapy and treatment protocols employed to improve the management of cancer patients. The journal also focuses on the impact of management programs and new therapeutic

Submit your manuscript here: https://www.dovepress.com/oncotargets-and-therapy-journal agents and protocols on patient perspectives such as quality of life, adherence and satisfaction. The manuscript management system is completely online and includes a very quick and fair peer-review system, which is all easy to use. Visit http://www.dovepress.com/ testimonials.php to read real quotes from published authors. 